\title{
LA PRECARIEDAD TE HACE DÓCIL. PROBLEMAS QUE AFECTAN A LA PROFESIÓN PERIODISTIICA
}

\author{
Mònica Figueras-Maz, Marcel Mauri-Ríos, Salvador Alsius-Clavera y Francesc \\ Salgado-De-Dios
}

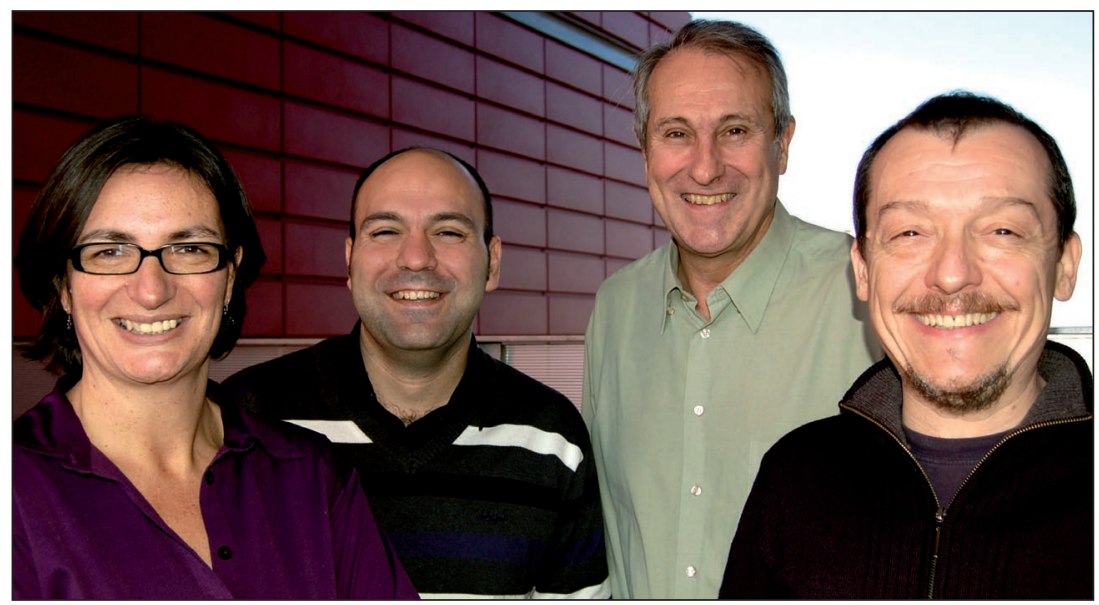

Mònica Figueras-Maz es doctora en periodismo por la Universitat Pompeu Fabra de Barcelona (UPF) y licenciada en ciencias de la información por la misma universidad. Trabaja desde 1998 como profesora en el Departamento de Comunicación de la UPF. Su línea de investigación se basa en los aspectos deontológicos de la comunicación, especialmente en relación con la juventud, el género y la inmigración.
Universitat Pompeu Fabra Campus de la Comunicació - Poblenou Roc Boronat, 138. 08018 Barcelona monica.figueras@upf.edu

Marcel Mauri-Ríos es doctor en periodismo por la Universitat Pompeu Fabra de Barcelona (UPF). Sus principales líneas de investigación son la historia del periodismo y la deontología periodística. Imparte docencia en el Departamento de Comunicación de la UPF y en los estudios de comunicación audiovisual de la Universitat Oberta de Catalunya (UOC). Es miembro del Grup de Recerca en Periodisme. Ha realizado estancias de investigación predoctorales en la Université Paris // y postdoctoral en la Columbia University de Nueva York.

Universitat Pompeu Fabra Campus de la Comunicació - Poblenou Roc Boronat, 138. 08018 Barcelona marcel.mauri@upf.edu

Salvador Alsius-Clavera es profesor titular de periodismo audiovisual en la Universitat Pompeu Fabra (UPF) y actualmente dirige la carrera de periodismo en dicha universidad. Es doctor en periodismo, licenciado en ciencias económicas y en ciencias de la información. Pertenece al Grupo de Investigación en Periodismo (GRP) y es el investigador principal del equipo que trabaja en ética de la información. A lo largo de su carrera ha alternado o compaginado una dedicación intensa a la actividad periodística (en prensa, radio y muy especialmente en televisión) con la docencia, la investigación y la gestión universitaria. Fue decano del Colegio de Periodistas de Catalunya y actualmente es miembro del Consejo de la Información de Catalunya.

Universitat Pompeu Fabra Campus de la Comunicació - Poblenou Roc Boronat, 138. 08018 Barcelona salvador.alsius@upf.edu

Francesc Salgado-De-Dios es profesor de la facultad de Comunicación de la Universitat Pompeu Fabra de Barcelona (UPF), en la que se doctoró en 2009 con una tesis sobre Manuel Vázquez Montalbán. Imparte cursos de redacción e historia del periodismo. Investiga la ética periodística y la historia de la prensa contemporánea. Licenciado en filosofía y ciencias de la educación por la Universitat de Barcelona (UB) y en periodismo por la Universitat Pompeu Fabra (UPF).

Universitat Pompeu Fabra Campus de la Comunicació - Poblenou Roc Boronat, 138. 08018 Barcelona francesc.salgado@upf.edu

\section{Resumen}

Se muestran los problemas que son percibidos como los más importantes por los periodistas de cuatro comunidades autónomas españolas: Catalunya, Madrid, País Vasco y Andalucía. De acuerdo con los resultados obtenidos en un trabajo de campo que mezcla metodología cuantitativa y cualitativa, la precariedad y la inseguridad laboral son los problemas que más preocupan a los periodistas de todas las comunidades consultadas. Las variables, edad, sexo e ideología son las que ofrecen más diferencias en las respuestas.

Artículo recibido el 26-07-11

Aceptación definitiva: 16-11-11 


\section{Palabras clave}

Ética periodística, Problemas de la profesión, Periodistas, Comunicación, Precariedad laboral, Intrusismo, Intereses económicos y políticos, España.

Title: Insecurity makes you docile. Perception of problems facing journalists

\section{Abstract}

The problems that are perceived as the most important by journalists from four Spanish regions: Catalonia, Madrid, Basque Country and Andalusia are reported. Fieldwork using both quantitative and qualitative methods found that precarious and uncertain job security are the issues of most concern to journalists in all the regions surveyed. Of the variables analyzed, age, sex and ideology accounted for the greatest differences in responses.

\section{Keywords}

Media ethics, Professional issues, Journalists, Professional precarity, Intrusion, Economic and political interests, Spain.

Figueras-Maz, Mònica; Mauri-Ríos, Marcel; Alsius-Clavera, Salvador; Salgado-De-Dios, Francesc. "La precariedad te hace dócil. Problemas que afectan a la profesión periodística". El profesional de la información, 2012, enero-febrero, v. 21 , n. 1 , pp. $70-75$.

http://dx.doi.org/10.3145/epi.2012.ene.09

\section{Introducción}

La crisis económica global ha afectado de forma muy clara a los medios de comunicación, y esta circunstancia, sumada a la imparable revolución tecnológica-comparable a la que cinco siglos antes se produjo con la llegada de la imprenta-, ha traído profundos cambios a la industria periodística (Díaz-Noci, 2010, p. 561). Varios trabajos han demostrado cómo desde 2009 las empresas periodísticas se han visto inmersas en una espiral de pérdidas económicas, concentración de medios y cierre de proyectos (Almiron, 2009), en un momento en que el trabajo de las redacciones tradicionales está siendo desafiado y cuestionado en buena parte por una nueva era de ciudadanos-productores de noticias (Compton; Benedetti, 2010).

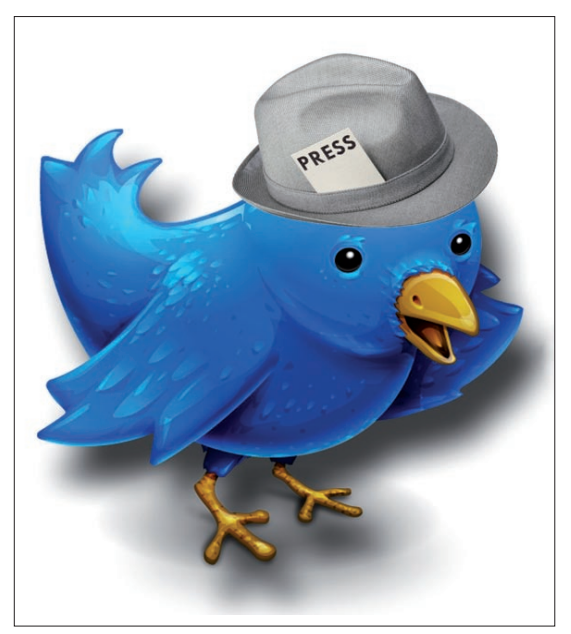

El principal objetivo de este artículo es mostrar los problemas de la profesión periodística a partir de un estudio empírico que analiza en profundidad la percepción de los propios periodistas de cuatro comunidades autónomas de España: Catalunya, País Vasco, Madrid y Andalucía ${ }^{1}$. Esta investigación tiene como principal antecedente el Libro blanco de la profesión periodística en Cataluña (2006), aunque sólo se hiciera en esta comunidad y con otra finalidad. También cabría señalar el Informe anual de la profesión periodística de la Asociación de la Prensa de Madrid (APM) y el Libro blanco de la prensa diaria elaborado por la Asociación Española de Editores de Diarios (AEDE), estudios que contemplan algunos de los problemas profesionales aunque no se ciñen exclusivamente a estos asuntos (Maciá-Barber; Herrera-Damas, 2010).

Fuera de España destacan los estudios de Noordenstreng y Hannikainen (1984) sobre la deontología y su aplicación en la profesión periodística en Finlandia y Europa, y de ClaudeJean Bertrand (2000, p. 108), pionero en la introducción en el ámbito de la comunicación de los estudios sobre los sistemas de rendición de cuentas de los medios (media accountability systems). En los Estados Unidos el estudio de referencia es The American journalist, de David Weaver y Cleveland Wilhoit (1986), actualizado en varias ocasiones, la última en 2007. Con su investigación estos autores realizan un retrato fidedigno de la evolución de la profesión periodística, sus valores y condiciones laborales en Estados Unidos, que es extrapolable al mundo occidental. Cabe señalar la contribución de Susanne Fengler y Stephan RussMohl (2008) acerca del comportamiento del periodista ante los retos y contextos profesionales contemporáneos. Son también muchas las investigaciones recientes sobre los condicionantes políticos (Hallin; Mancini, 2004), los imperativos económicos que afectan a la tarea de los periodistas en su trabajo tradicional (Benson; Hallin, 2007; McManus, 2009) o la convergencia que provoca internet (Casero-Ripollés, 2010). El análisis desde la perspectiva de los propios periodistas lo encontramos en Hanitzsch y Mellado (2011), que comparan el grado de presiones e influencias que perciben en el trabajo de periodistas de 18 países, entre ellos España. Lo hacen mediante una encuesta llevada a cabo en un trabajo previo (Hanitzsh et al., 2010) que contempla seis dimensiones (influencias políticas, económicas, organizacionales, profesionales, de procedimiento y de grupos de referencia). Los factores políticos y económicos son claramente los denominadores más importantes en las diferencias nacionales: así, los españoles, junto con los australianos, perciben influencias políticas y económicas, a diferencia del resto de periodistas de países occidentales que no lo sienten así. 


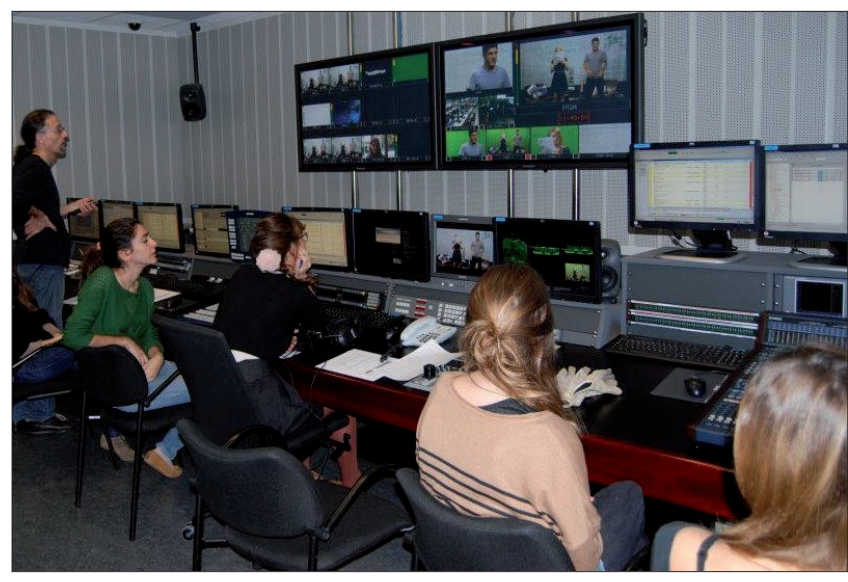

Sala de control de una televisión

De todos los problemas planteados, la cuestión que más preocupa a los profesionales es la precariedad y la inseguridad laboral

\section{Metodología aplicada}

Para el estudio que presentamos en este artículo se tomó como punto de partida un análisis de los códigos y documentos con normativa deontológica existentes en España, a partir de los trabajos de compilación de textos realizados por Hugo Aznar (1999) y Juan-Carlos Pérez-Fuentes (2004) que sirvió de marco teórico para la investigación: un análisis cualitativo (entrevistas en profundidad) y cuantitativo (encuestas online) para conocer los criterios éticos interiorizados por los profesionales del periodismo. El trabajo de campo (entrevistas en profundidad y encuesta online) se delegó en empresas expertas en investigación de mercados o, en el caso de Catalunya y Madrid, en los colegios profesionales de sociólogos.

El número de entrevistas en cada comunidad autónoma varió en función del censo de periodistas: en Madrid y Catalunya se efectuaron 30, en el País Vasco 20, y en Andalucía 24. Se llevaron a cabo entre abril y junio de 2008 y los resultados obtenidos fueron utilizados como punto de partida para diseñar la encuesta online compuesta por 43 preguntas. Esta fase del trabajo de campo tuvo lugar a lo largo de tres semanas a finales de 2008 y principios de 2009. El número de encuestas obtenidas fue de 2.012, repartidas de la siguiente manera: Catalunya, 1.198; Madrid, 417; País Vasco, 172; Andalucía, 225.

Para realizar los cuestionarios se eligieron los temas más candentes recogidos por los códigos éticos, libros de estilo, estatutos de redacción y recomendaciones específicas de instituciones autorreguladoras: defensores de los lectores, consejos de la información, etc. También se recogieron los problemas estructurales y coyunturales que tiene el sistema periodístico español, en la medida que forma parte del modelo mediterráneo o pluralista polarizado -en el que la influencia política y el partidismo de la prensa es muy importante- según la definición que hacen Daniel Hallin y Paolo
Mancini (2004, pp. 89). Precisamente, en otro estudio de Hallin y Papathanassopoulos (2002) se concluye que existe un alto nivel de percepción de influencia política en los países con fuertes tradiciones de pluralismo polarizado, como el sur de Europa y América Latina.

En este artículo se desgranan los resultados de una de las preguntas de la encuesta online, la que se refería a los que posiblemente son percibidos como principales problemas de la profesión periodística, a la vez que se explotan las respuestas que se obtuvieron de las entrevistas en profundidad.

La pregunta se formuló de la siguiente forma: ¿Cuál es su percepción sobre los siguientes problemas que supuestamente afectan a la profesión periodística? Y se planteaban ocho ítems:

- precariedad laboral,

- concentración de medios,

- influencia de los índices de audiencia,

- predominio de los intereses políticos y económicos por encima de los periodísticos,

- falta de ética profesional,

- confusión de tareas de otras profesiones y el intrusismo profesional,

- bajo respeto hacia la independencia de los profesionales, y

- falta de conciencia hacia la responsabilidad social de los medios.

No hay grandes diferencias entre la opinión de hombres y mujeres, salvo en el caso del intrusismo

\section{Precariedad e inseguridad como principales problemas de la profesión}

De los problemas planteados, la cuestión que más preocupa a todos los periodistas encuestados es la precariedad y la inseguridad laboral. El segundo aspecto que suscita más inquietud es el predominio de los intereses económicos y políticos sobre los periodísticos. En la fase cualitativa estos dos temas son también recurrentes.

Los profesionales andaluces aparecen especialmente sensibilizados con estos dos problemas y no dudan en considerar la profesión como "una selva donde muchos mercenarios trabajan gratis o por muy poco dinero". Asimismo, consideran que la inseguridad laboral condiciona la calidad y la libertad de expresión porque "el periodista precario es más dócil". La novedad en el discurso generalizado está en Catalunya, donde 8 de las 30 personas consultadas encuentran "lógica" y "admisible" y en algún caso hasta positiva la influencia que ejercen los intereses económicos y políticos en el periodismo.

Tras esos dos problemas, la influencia de los índices de audiencia, la falta de ética profesional y la baja conciencia de la responsabilidad social del periodista son consideradas preocupaciones bastante o muy importantes en todas las co- 
munidades analizadas (más de 8 de cada 10 encuestados). La excepción son Andalucía y el País Vasco. En la comunidad andaluza hay una preocupación ligeramente inferior por los índices de audiencia (76,8\%); y los periodistas vascos se muestran menos afectados por la baja conciencia de la responsabilidad social del periodista, con un $75 \%$ de respuestas positivas. Sin embargo, la concentración de los medios preocupa especialmente en el País Vasco y los entrevistados de esta comunidad reconocen que no tienen otra opción que plegarse a las condiciones impuestas por las empresas.

El intrusismo profesional y la confusión con las tareas propias de otras profesiones, si bien son temas considerados importantes para más de la mitad de los comunicadores, representan una inquietud menor. En ambos casos, los más preocupados son los vascos y los menos, los madrileños. En un término medio se sitúa la preocupación por la falta de respeto a la autonomía de los profesionales y, nuevamente, los vascos $(90,1 \%)$ son los que tienen una percepción superior del problema y los madrileños, menor (76,6\%). Las entrevistas en profundidad en el País Vasco refuerzan esta impresión: "en este sector ha entrado cualquiera que tuviese unas mínimas dotes comunicativas".

En la tabla 1 pueden apreciarse las diferencias y similitudes en las respuestas dadas por los profesionales consultados.

\section{Diferencias de percepción según variables}

Mientras que en Andalucía y el País Vasco no se detectan diferencias significativas según variables independientes, en Catalunya y Madrid las respuestas varían en función de la edad, sexo, ingresos e ideología de los encuestados. En el caso catalán, condiciona el medio de trabajo y en Madrid lo hacen el nivel de estudios, el tamaño de la empresa, la categoría profesional y el área temática.

\section{Los de mayor edad y los de izquierdas, los más pre- ocupados}

Respecto a la edad, se produce una relación directamente proporcional con la importancia otorgada a los problemas, de manera que cuanto mayor es la persona más importancia concede a los factores planteados. Los mayores de 50 años manifiestan mayor inquietud por la falta de respeto a la autonomía de los profesionales, y por la concentración de medios. Esto se invierte en el caso de la precariedad labo- ral y el intrusismo profesional: son los menores de 35 años los más sensibilizados con este problema. Por ejemplo, el $73,3 \%$ de los profesionales catalanes menores de 35 años lo considera muy importante, mientras que sólo preocupa al $58,6 \%$ de los de otras edades. La franja de menor edad de este territorio también es la que más se preocupa por los índices de audiencia.

La ideología aparece así mismo como una variable que refleja diferencias: los comunicadores que se declaran de izquierdas son los que muestran mayor grado de inquietud respecto a la práctica totalidad de los problemas planteados. La excepción a esta tendencia se produce en Catalunya y respecto al problema de la falta de ética profesional: los profesionales catalanes de centro y derecha conciben el problema de forma ligeramente superior $(37,6 \%$ frente al $31,6 \%$ de izquierda).

\section{Los profesionales de izquierda muestran mayor grado de inquietud respecto a los problemas planteados}

\section{Ellas, más sensibilizadas con el intrusismo que ellos}

No hay grandes diferencias entre la opinión de los hombres y mujeres consultados, salvo en el caso del intrusismo. Las periodistas están más sensibilizadas por esta cuestión que los hombres. En el caso catalán, esto se constata con un $71,8 \%$ y un $51,4 \%$ de respuestas positivas respectivamente. En Madrid, 7 de cada 10 mujeres lo consideran un problema bastante o muy importante frente a casi 5 de cada 10 hombres. También en esta comunidad, el predominio de los intereses económicos y políticos sobre los periodísticos preocupa más a los hombres que a las mujeres.

\section{El intrusismo preocupa más a los licenciados en pe- riodismo y a los trabajadores de empresas públicas}

Por nivel de estudios, el único supuesto que presenta diferencias notables es el del intrusismo profesional. Esto se debe a que los licenciados en periodismo -en un entorno creciente de periodismo ejercido por no licenciados o ciudadanos-productores- perciben el problema de forma más im-

\begin{tabular}{|c|c|c|c|c|}
\hline & Madrid & Catalunya & Andalucía & País Vasco \\
\hline Precariedad laboral & 93,7 & 95,0 & 97,8 & 96,5 \\
\hline Concentración de medios & 70,5 & 74,8 & 75,9 & 90,1 \\
\hline Índices de audiencia & 83,6 & 81,2 & 76,8 & 89,5 \\
\hline Intereses económicos y políticos & 90,0 & 92,9 & 95,1 & 93,1 \\
\hline Falta de ética profesional & 84,9 & 82,1 & 87,4 & 83,7 \\
\hline Confusión de tareas de otras profesiones & 52,9 & 57,0 & 60,6 & 77,9 \\
\hline Intrusismo profesional & 54,4 & 60,1 & 69,1 & 79,7 \\
\hline Falta de respeto a la autonomía de los profesionales & 76,6 & 79,8 & 81,8 & 90,1 \\
\hline Baja conciencia de la responsabilidad social del periodista & 81,0 & 81,0 & 86,7 & 75,0 \\
\hline
\end{tabular}

Tabla 1. Problemas percibidos como "bastante" o "muy" importantes (\%). Las columnas están ordenadas de menor (Madrid) a mayor (País Vasco) intensidad problemática. Los números en rojo marcan los valores máximos de las respuestas 
portante que los que no lo son. Los andaluces entrevistados en la fase cualitativa creen que no hay forma de acabar con el intrusismo, al cual se le considera la lacra actual. Algunos dicen que se debe restringir el acceso a los que no son licenciados; en cambio otros no dudan en afirmar que se puede hacer muy buen periodismo sin ser licenciado. También se aprecian algunas diferencias, sobre todo en Catalunya, entre trabajadores de empresas públicas y privadas -más sensibles a esta cuestión los primeros que los segundos- ante la preocupación por el intrusismo.

\section{Los ingresos, la situación y la categoría laboral mar-} can algunas diferencias

Se observa que a mayores ingresos, menor preocupación por los diferentes problemas. Por ejemplo, los profesionales catalanes que cobran más de 3.000 euros mensuales son los que menos inquietud muestran por la concentración de los medios de comunicación, la confusión con las tareas propias de otras profesiones, la falta de ética profesional, el intrusismo y la falta de autonomía profesional.

La preocupación por la precariedad y la inseguridad laboral también se reduce a medida que aumenta el salario. Los profesionales que se encuentran sin trabajo, autónomos y asalariados conceden mayor importancia a este asunto que los empresarios con trabajadores. Del mismo modo, los redactores o equivalentes conceden más importancia a este problema que los jefes de sección o los directivos, por lo que se puede deducir que a mayor jerarquía menor importancia se concede al problema. En la fase cualitativa se constató que los cargos intermedios, quienes se ven en la posición de transmitir las presiones, parecen haberlas internalizado o asumido, de manera que podrían ser menos conscientes de su gravedad. Por el contrario, los trabajadores que ocupan posiciones inferiores en la jerarquía de sus empresas expresan con mayor frecuencia su incomodidad respecto de la falta de autonomía en el desempeño de su trabajo cotidiano.

En el País Vasco no hay tanta preocupación por la conciencia de la responsabilidad social y en cambio, mucha por la falta de autonomía profesional

\section{Conclusiones}

La situación económica mundial y española, de la que la industria periodística no se escapa, afecta de forma directa a los profesionales de la información. Prueba de ello es que la precariedad e inseguridad laboral son los principales problemas aludidos por los periodistas. Que más de un $90 \%$ de los encuestados señalen esta cuestión como problemática demuestra el grado de inestabilidad laboral de los periodistas, y más si se tiene en cuenta que el estudio se hizo en 2008 y 2009 , cuando la crisis todavía no había mostrado toda su dureza.

La segunda preocupación más señalada, el predominio de los intereses económicos y políticos sobre los periodísticos, es compartida por más del $90 \%$ de los profesionales encuestados, que ponen de manifiesto la vulnerabilidad de la información ante los poderes políticos y/o financieros.

En general no hay diferencias significativas por sexos pero resulta interesante observar cómo, en el caso de la precariedad, las mujeres se preocupan más que los hombres: ellas tienen mayor sensación de vulnerabilidad en sus puestos de trabajo que ellos. También se sienten más inseguros laboralmente los jóvenes periodistas, un hecho que parece lógico en un momento de crisis de medios y de difícil acceso al mercado laboral. En cambio, cuanto más mayor se es, más preocupación existe por el resto de supuestos de componente más ético.

En el País Vasco y en Andalucía, la confusión de tareas profesionales y el intrusismo preocupan bastante más que en Madrid y Catalunya

Los resultados son bastante parecidos cuando se comparan los cuatro territorios analizados. En general, los índices de audiencia, la falta de ética profesional y la baja conciencia de la responsabilidad social del periodista aparecen como preocupaciones bastante o muy importantes en todas las comunidades analizadas. Pero los resultados no son tan transversales cuando se trata de problemas laborales concretos. Así, en el País Vasco no hay tanta preocupación por la conciencia de la responsabilidad social y en cambio mucha por la falta de autonomía profesional, quizá debido a la sensación de presión al trabajar en un entorno acosado por el terrorismo. Sería, pues, interesante observar si los resultados pueden cambiar a partir de ahora con el abandono de las armas de ETA. También hay claras diferencias en la preocupación por la concentración de medios, 20 puntos más en el País Vasco que en Madrid, un diferencial que puede encontrar explicación en el censo de profesionales mucho más reducido en el País Vasco, y en el temor a ser absorbidos por grupos empresariales con sede fuera de esta comunidad y, con ello, la posible pérdida de la idiosincrasia local.

Finalmente, cabe destacar que en el País Vasco y en Andalucía, la confusión de tareas profesionales y el intrusismo preocupan bastante más que en Madrid y Catalunya. Parece que en entornos donde el censo de periodistas es menor, se es más sensible a un periodismo amateur muy vinculado a las nuevas tecnologías de la información: cualquier ciudadano con un smartphone puede compartir información. En futuras encuestas sobre la profesión se podrá ver si esto es sólo una cuestión coyuntural (debido, también, a la crisis) o no.

\section{Nota}

1. Esta investigación es resultado del proyecto "Ética y excelencia informativa. La deontología periodística frente a las expectativas de los ciudadanos", no de referencia SEJ 200605631-C05-01, del Plan Nacional de Investigación Científica, Desarrollo e Innovación Tecnológica 2004-2007 del Ministerio de Educación y Ciencia. 


\section{Bibliografía}

Almiron, Núria. "Grupos privados propietarios de medios de comunicación en España: principales datos estructurales y financieros". Comunicación y sociedad, 2009, v. 22, n. 1, pp. 243-273.

http://www.unav.es/fcom/comunicacionysociedad/es/ articulo.php?art_id $=36$

Aznar, Hugo. Ética y periodismo: autorregulación, códigos, estatutos de redacción y otros documentos. Barcelona: Paidós, 1999. ISBN 8449306531

Benson, Rodney; Hallin, Daniel. "How states, markets and globalization shape the news. The French and US national press, 1965-97". European journal of communication, 2007, v. 22 , n. 1 , pp. $27-48$.

http://dx.doi.org/10.1177/0267323107073746

Bertrand, Claude-Jean. Media ethics \& accountability systems. New Brunswick: Transaction Publishers, 2000. ISBN 1560004207

Casero-Ripollés, Andreu. "Prensa en internet: nuevos modelos de negocio en el escenario de la convergencia". El profesional de la información, 2010, v. 19, n. 6, pp. 595-601. http://eprints.rclis.org/bitstream/10760/15015/1/595-601. $p d f$

http://dx.doi.org/10.3145/epi.2010.nov05

Compton, James R.; Benedetti, Paul. "Labour, new media and the institutional restructuring of journalism". Journalism studies, 2010, v. 11, n. 4, pp. 487-499.

http://dx.doi.org/10.1080/14616701003638350

Díaz-Noci, Javier. "Medios de comunicación en internet: algunas tendencias". El profesional de la información, 2010, v.19, n. 6, pp. 561-567.

http://www.elprofesionaldelainformacion.com/conteni dos/2010/noviembre/medios_comunicacion.pdf

http://dx.doi.org/10.3145/epi.2010.nov.01

Fengler, Susanne; Russ-Mohl, Stephan. "Journalists and the information-attention markets: towards an economic theory of journalism". Journalism, 2008, v. 9, n. 6, pp. 667690.

http://www.journalistik-dortmund.de/fileadmin/content/ fengler/Journalists_and_Information-Attention-Markets.pdf http://dx.doi.org/10.1177/1464884908096240

Hallin, Daniel; Mancini, Paolo. Comparing media systems: three models of media and politics. Cambrigde: Cambridge University Press, 2004. ISBN 0521543088
Hallin, Daniel; Papathanassopoulos, Stylianos. "Political clientelism and the media: Southern Europe and Latin America in comparative perspective". Media, culture \& society, 2002, v. 24, n. 2, pp. 175-95.

http://www.portalcomunicacion.com/both/opc/client.pdf

Hanitzsch, Thomas; Mellado, Claudia. "What shapes the news around the world? How journalists in eighteen countries perceive influences on their work". International journal of press/politics, 2011, v. 16, n. 3, pp. 404-426. http://dx.doi.org/10.1177/1940161211407334

Hanitzsch, Thomas; Anikina, Maria; Berganza, Rosa; Cangoz, Incilay; Coman, Mihai; Hamada, Basyouni; Hanusch, Folker et al. "Modeling perceived influences on journalism: evidence from a cross-national survey of journalists". Journalism \& mass communication quarterly, 2010, v. 87, n. 1, pp. 7-24.

Maciá-Barber, Carlos; Herrera-Damas, Susana. "La deontología periodística: praxis, disfunciones y retos desde la perspectiva de los profesionales de la comunicación en la Comunidad de Madrid (2006-2009)". Comunicación y sociedad, 2010, v. 23, n. 1, pp. 77-104.

http://www.unav.es/fcom/comunicacionysociedad/es/ articulo.php?art_id=351

McManus, John H. "The commercialization of news". En: Wahl-Jorgensen, Karin; Hanitzsch, Thomas. The handbook of journalism studies. New York: Routledge, 2009, pp. 218235. ISBN 0203877683

Noordenstreng, Kaarle; Hannikainen, Lauri. The mass media declaration of Unesco. Norwood: Ablex, 1984. ISBN 0893910775

Pérez-Fuentes, Juan-Carlos. Ética periodística: principios, códigos deontológicos y normas complementarias. Bilbao: Servicio Editorial de la Universidad del País Vasco, 2004. ISBN 8483735946

Real-Rodríguez, Elena. "La autoregulación. Valoración de los códigos. Conocimiento de los mecanismos de autoregulación". En: Alsius, Salvador; Salgado, Francesc (eds.). La ética informativa vista por los ciudadanos. Barcelona: UOC, 2010, pp. 275-291. ISBN 9788497889353

Russ-Mohl, Stephan. "Regulating self-regulation: the neglected case of journalism policies". Communication and medicine, 1993, v. 18, n. 2, pp. 151-168.

Weaver, David; Wilhoit, Cleveland. The American journalist. Indianapolis: Indiana University Press, 1991 (2 ${ }^{\mathrm{a}}$ ed.). ISBN 0253363640

\section{Suscripción EPI sólo online}

Pensando sobre todo en los posibles suscriptores latinoamericanos, ya no es obligatorio pagar la suscripción impresa de EPI para acceder a la online.

EPI se ofrece a instituciones en suscripción "sólo online" a un precio considerablemente más reducido (99 euros/año), puesto que en esta modalidad no hay que cubrir los gastos de imprenta ni de correo postal. 


\section{masmedios}

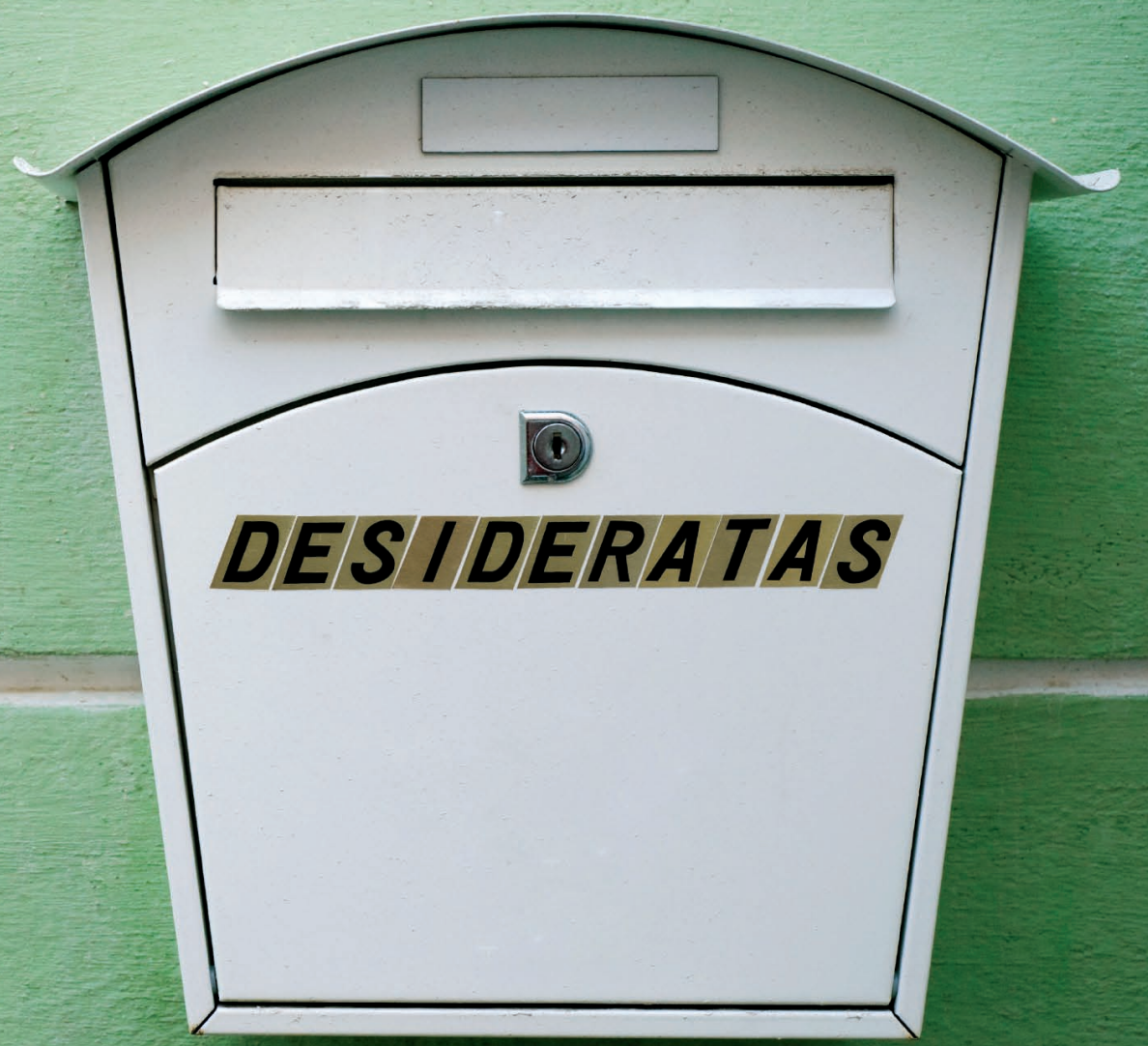

\section{Te entendemos 99}

Sistemas de gestión de bibliotecas Open Source Interfaces interactivas y OPACs Repositorios OAI

Gestión documental y de archivos Digitalización

Outsourcing de servicios documentales Desarrollo de sitios web / multimedia / e-learning Comunicación y e-marketing de servicios de información 